\title{
Cuidados dermocosméticos no tratamento da rosácea: uma revisão da literatura sobre as perspectivas mundiais
}

\author{
Dermocosmetic care in the treatment of rosea: a review of the literature on world \\ perspectives
}

Míria Dantas Pereira ${ }^{1 *}$, Mara Dantas Pereira²

${ }^{1}$ Farmacêutica. Graduada em Farmácia pela Universidade Tiradentes; ${ }^{2}$ Graduada em Psicologia pela Universidade Tiradentes.

\begin{abstract}
Resumo
Introdução: a rosácea é uma doença inflamatória de pele, de etiologia desconhecida. Apresenta-se na região centro-facial, com a presença de rubor, eritema, telangiectasias, pápulas e pústulas, periódicas ou persistentes. Objetivo: o objetivo deste trabalho é realizar uma revisão bibliográfica narrativa de estudos envolvendo os cuidados dermocosméticos no tratamento da rosácea. Metodologia: as buscas foram realizadas nas bases de dados: Biblioteca Virtual em Saúde (BVS), Scientific Electronic Library Online (SciELO), Literatura Latino-americana e do Caribe em Ciências da Saúde (LILACS) e PubMed. A pesquisa envolveu artigos de revistas e periódicos, monografias e dissertações em português, inglês e francês, publicadas entre os anos de 2014 a 2020. Resultados: a pele dos pacientes com rosácea é suscetível a irritabilidade, logo, é necessário uma série de cuidados diários com a pele desde a limpeza, hidratação, proteção solar, que possibilitam efeitos calmantes que melhoram a qualidade da pele. Conclusão: devido à natureza multifatorial da doença não há tratamento padronizado. Logo, os profissionais envolvidos nos cuidados desses pacientes devem esclarecer aos pacientes sobre as ações e cuidados necessários para o uso das terapêuticas, incluindo os dermocosméticos. Palavras - chave: Rosácea. Dermocosméticos. Cuidados de Pele. Controle da rosácea.
\end{abstract}

\begin{abstract}
Introduction: rosacea, erythema, telangiectasias, papules and pustules, periodic or persistent. Objective: the objective of this work is to carry out a narrative bibliographic review of studies involving dermocosmetic care in the treatment of rosacea. Methodology: Searches were carried out in the databases: Virtual Health Library (VHL), Scientific Electronic Library Online (SciELO), Latin American and Caribbean Literature in Health Sciences (LILACS) and PubMed. The research involved magazine and periodical articles, monographs and dissertations in Portuguese, English and French, published between 2014 and 2020. Results: the skin of patients with rosacea is susceptible to irritability, therefore, a series of daily care is necessary with the skin from cleansing, hydration, sun protection, which enable calming effects that improve the quality of the skin. Conclusion: due to the multifactorial nature of the disease, there is no standardized treatment. Therefore, the professionals involved in the care of these patients must inform patients about the actions and care necessary for the use of therapies, including dermocosmetics.

Keywords: Rosacea. Dermocosmetics. Skin care. Rosacea Control.
\end{abstract}

\section{INTRODUÇÃO}

A rosácea é caracterizada como uma doença crônica cutânea comum. Apresentando como principais características, eritema centro-facial transitório ou persistente, telangiectasias, pápulas e pústulas inflamatórias, bem como sintomas oculares e alterações fimatosas (KARAOSMANOGLU; KARAASLAN; CETINKAYA, 2020).

A rosácea é um distúrbio dermatológico que atinge cerca de 5-10\% da população mundial (BALDWIN et al., 2019). No Brasil, de acordo com dados divulgados pela Sociedade Brasileira de Dermatologia (2018), estima-se que cerca $1,5 \%$ de indivíduos possuem a doença no país.

Correspondente/Corresponding: *Míria Dantas Pereira-Departamento de Farmácia da Universidade Tiradentes. - End: Avenida Murilo Dantas, 300, Campus Farolândia - Aracaju - Sergipe - Brasil, CEP 49032-490. Tel: (79) 3218-2100. - E-mail: miriadantaspereira@gmail.com

Rev. Ciênc. Méd. Biol., Salvador, v. 19, n. 2, p. 361-366, mai./ago. 2020
Observa-se que assim como outras doenças crônicas, a rosácea é tratável em vez de curável e requer intervenção a longo prazo. Em condições leves, o tratamento com dermocosméticos pode ser uma excelente alternativa. Podendo ser utilizada como única terapêutica ou como adjuvante de outras terapias, devido à ausência de riscos de eventos adversos, interações medicamentosas e resistência a antibióticos (SIDDIQUI; GOLD; GILL, 2016).

A palavra "cosmético" é derivada do grego "Kosmetikos", tendo como significados "adornar e embelezar". O conceito de beleza e aparência sempre representou uma parte integrante do ser humano, desde os primeiros registros de seu uso encontrados no Egito (1400 a.C.) (GONÇALVES; PINA, 2017).

Nesse contexto, a Agência Nacional de Vigilância Sanitária (ANVISA) (2015), publicou a RDC no 07/2015, que define os cosméticos como preparações constituídas por 
substâncias naturais ou sintéticas, para uso externo em diversas partes do corpo, tais como, pele, sistema capilar, etc., com o objetivo de limpá-los, perfumá-los, alterar sua aparência, protegê-los ou mantê-los em bom estado.

Nos últimos anos, com avanços científicos e investimentos de recursos em estudos na área da beleza, foi possível a criação de produtos inovadores, tais como, os chamados dermocosméticos. Produtos caracterizados por trazerem ativos farmacológicos em sua composição, tendo foco na beleza, mas também na saúde da pele. No Brasil, o termo dermocosméticos ainda não é reconhecido, sendo classificados como cosméticos tipo 2 (GONÇALVES; PINA, 2017).

Logo, a Agência Nacional de Vigilância Sanitária (ANVISA) (2015), através da RDC no 07/2015, define cosméticos tipo 2 como produtos que em sua formulação devam possuir indicações específicas, e as características exigem comprovação de segurança e eficácia, assim como informações e cuidados, modo e restrições de uso.

O objetivo deste trabalho é realizar uma revisão bibliográfica narrativa de estudos envolvendo o uso de dermocosméticos no tratamento da rosácea, e reconhecer quais são os cuidados gerais dermocosméticos para o tratamento e a estabilização desta doença.

\section{METODOLOGIA}

Para o desenvolvimento do presente trabalho, realizou-se uma revisão narrativa da literatura. A investigação bibliográfica foi realizada entre o período de setembro de 2019 até janeiro de 2020. Por meio da busca de artigos de revistas e periódicos, monografias e dissertações publicados nos idiomas de português, inglês e francês disponíveis nas seguintes bases de dados: Biblioteca Virtual em Saúde (BVS), Scientific Eletronic Library Online (SciELO), Literatura Latino-americana e do Caribe em Ciências da Saúde (LILACS) e PubMed.

Para tal, foram utilizados os seguintes Descritores em Ciências da Saúde (DeCS): "Rosácea”, "Dermocosméticos", "Cuidados de Pele" e "Controle da Rosácea". Estes termos encontram-se no DeCS, respectivamente, da seguinte forma em inglês: "Rosacea"; "Dermocosmetics"; "Skin care"; "Control of rosacea".

Utilizou-se o MeSH Database "Medical Subject Headings", como estratégia de busca: ("Rosacea" [Mesh]) AND "Dermocosmetics" [Mesh], juntamente com operador boleano "AND", utilizando de modo combinado ou puro por meio da utilização dos demais descritores. Sendo encontrados 261 publicações.

Posteriormente, foram utilizados os seguintes critérios de inclusão: artigos científicos na íntegra, publicados entre os anos de 2014 a 2020, nos idiomas português, inglês e francês, os quais abordassem a temática discutida. Os critérios de exclusão estabelecidos compreenderam publicações, tais como: livros e resumos. Além de artigos publicados em modo retrativo com publicações anteriores ao ano de 2014 e que não fossem da língua portuguesa, inglesa e francesa, duplicados e que não estivessem na íntegra. Chegando-se ao total de 22 publicações selecionadas para o estudo (Figura 1).

Figura 1 - Fluxograma demonstrativo da metodologia desenvolvida no presente estudo

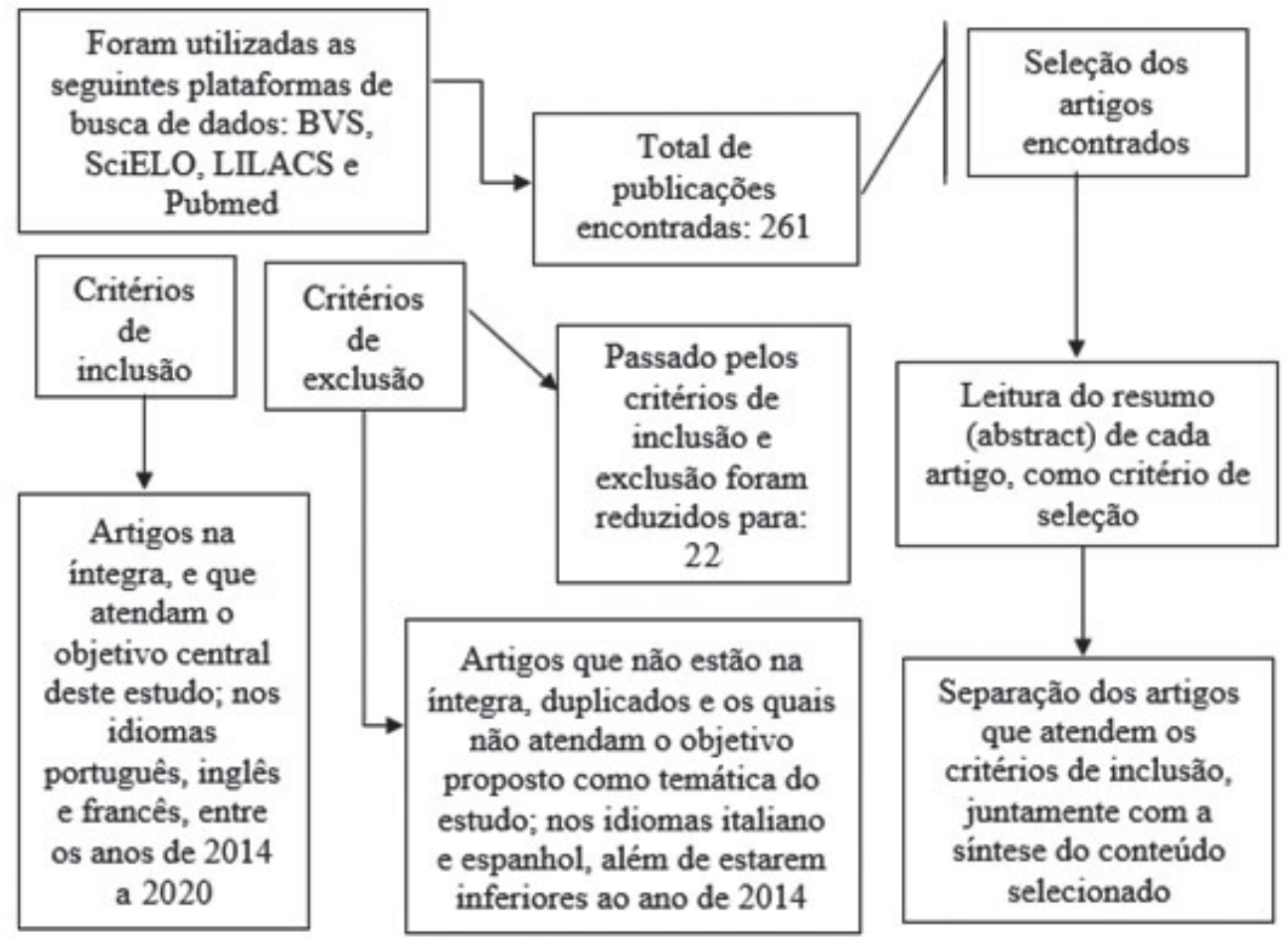

Fonte: Autoras (2020). 


\section{RESULTADOS E DISCUSSÃO}

\section{ROSÁCEA}

A rosácea é um distúrbio cutâneo inflamatório de natureza crônica. Onde a presença de uma ou mais características distribuídas nas áreas centro-faciais é indicativo da rosácea. Sinais e sintomas como, rubor, eritema transitório ou persistente, pápulas, pústulas e telangiectasias (vasos muito finos). As áreas mais afetadas são bochechas, nariz, queixo e testa (ERDOGAN et al., 2018).

Manifesta-se principalmente em indivíduos de fototipo I e II (classificação Fitzpatrick). Contudo, a rosácea também pode afetar indivíduos de peles mais escuras. $O$ início geralmente ocorre em adultos entre 30-50 anos de idade, porém pode ocorrer em qualquer período da vida, e com maior predominância de atingir o sexo feminino (FLOREZ-WHITE, 2019; VAN ZUUREN, 2017).

A rosácea apresenta várias características clínicas diferentes. Por tal motivo, em 2002, o Comitê de Especialistas da Sociedade Internacional de Rosácea introduziu um sistema classificatório que divide a rosácea em quatro subtipos: eritemato-telangiectásica, pápulo-pustulosa, fimatosa e ocular (SCHMUTZ, 2014).

Embora sua patogênese seja desconhecida, a causa do aparecimento da rosácea é presumivelmente um processo multifatorial. Distúrbios do sistema imune inato, mecanismos neuroinflamatórios, radiação UV, defeito da barreira cutânea, parecem desempenhar um papel de importância no processo fisiopatológico da doença
(BUDDENKOTTE; STEINHOFF, 2018; KRESKEN et al., 2018).

\section{CLASSIFICAÇÃO DA ROSÁCEA EM SUBTIPOS}

A rosácea é classificada em quatro subtipos: I - Rosácea eritemato-telangiectásica, II - Rosácea pápulo-pustulosa, III-Rosácea fimatosa e IV-Rosácea ocular (Figura 2) (SCHMUTZ, 2014).

Subtipo I - Rosácea eritemato-telangiectásica: Manifesta-se com o aparecimento de rubor, eritema persistente e telangiectasias, predominantes na região centro-facial (Figura 2.A). O rubor com mais de 10 minutos de duração, ajuda na diferenciação do rubor convencional (DEL ROSSO, 2014).

Subtipo II - Rosácea pápulo-pustulosa: É o segundo subtipo mais comum da doença. É caracteriza-se pelo eritema facial central persistente, além de pápulas e pústulas de natureza transitória (Figura 2.B) (ABOKWIDIR; FELDMAN, 2016; SCHMUTZ, 2014).

Subtipo III - Rosácea fimatosa: Este subtipo atinge mais os homens, e é caracterizado principalmente pelo alargamento do nariz, a partir de excesso de tecido e crescimento de glândulas sebáceas (Figura 2.C). Geralmente, há necessidade de cirurgia (KRESKEN et al., 2018; CRIBIER, 2017; ARAUJO, 2016).

Subtipo IV - Rosácea ocular (Figura 2.D): A rosácea também pode afetar a região dos olhos. Dentre os sintomas, incluem-se, sensação de corpo estranho, olhos secos, ardor, prurido e vermelhidão palpebral (ARAUJO, 2016; LUZURIAGA; MHLABA; ROMAN, 2016).

Figura 2 - Imagens representativas dos subtipos clínicos da rosácea
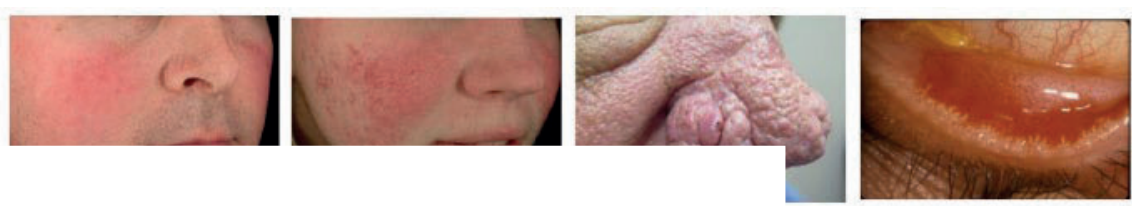

A) tritema b) rapuıas e pustuıas () rımatosa ע/ vcuıar

(A) Rosácea eritemato-telangiectásica, (B) Rosácea pápulo-pustulosa, (C) Rosácea fimatosa grave e (D) Rosácea ocular com infiltração de lipídios das glândulas de meibomianas que revestem a margem da pálpebra.

Fonte: Asai et al. (2016).

\section{DERMOCOSMÉTICOS PARA ROSÁCEA}

Atualmente, o mercado de dermocosméticos oferece uma ampla gama de produtos inovadores, produtos estes, que se diferenciam de outros itens cosméticos por conterem maiores concentrações de ativos. Dessa forma, os dermatologistas começaram a prescrever dermocosméticos não só para manter a aparência estética de seus pacientes, mas também para tratamento e cuidados de doenças inflamatórias de pele (DRENO et al., 2014).

Na rosácea, uma grande variedade de ingredientes ativos dermocosméticos (ex: bisabolol, alantoína, niacinamida, ácido hialurônico, etc.), que podem ser utilizados para fortalecer as paredes dos vasos sanguíneos, modular a vasomotricidade e diminuir o efeito perivascular do edema (DEL ROSSO, 2014).

Os pacientes com rosácea frequentemente se queixam de ter hipersensibilidade, intolerância a produtos de higiene e cosméticos. Esse desconforto chamado de "picada" (Tabela 1), faz cogitar uma alergia de contato, a diferentes produtos (conservantes, emulsionantes e perfumes). Estas peles sensíveis ou reativas necessitam de cuidados específicos, para purificar, nutrir e proteger a pele, levando a uma maior resistência da pele $(\mathrm{SCH}-$ MUTZ, 2014). 
Tabela 1 - Substâncias que induzem picadas cutâneas de intensidade leve e moderada

\begin{tabular}{l}
\hline Substâncias irritantes leves \\
\hline Benzeno \\
\hline Ácido salicílico \\
\hline Resorcina \\
\hline Substâncias irritantes moderadas \\
\hline Carbonato de Sódio \\
\hline Propilenoglicol \\
\hline Dimetilacetamida \\
\hline Peróxido de benzoíla \\
\hline
\end{tabular}

Fonte: Gonçalves e Pina (2017).

\section{LIMPEZA DE PELE}

Atualmente, existem vários tipos de produtos de limpeza facial, porém em uma pele com rosácea, deve-se evitar o uso de sabonetes faciais com sabão, pois estes são misturas de ésteres de ácidos gordos que produzem espuma e são alcalinos (pH 9,0-10,0), ou seja, aumentam o $\mathrm{pH}$ da pele, logo, podem destruir a camada lipídica superficial da pele, levando a uma secura excessiva da pele (GONÇALVES; PINA, 2017).

Deve-se também evitar o uso produtos de limpeza facial que contenham tensioativos aniônicos, como o lauril sulfato de sódio e o lauril éter sulfato de sódio, que possuem carga negativa, que têm forte poder espumante e detergente, o que não é indicado para uma pele sensível, pois retira a oleosidade natural da pele (SCHALLER et al., 2016).
É indicado priorizar o uso de sabonetes faciais, com um pH ligeiramente ácido, como é o caso dos Syndets (detergentes sintéticos) que possuem tensoativos anfotéricos (ex: sodium cocoamphoacetate), em sua composição, que promovem uma limpeza suave e com baixo potencial de irritabilidade. Outra opção são os fluidos de limpeza sem enxágue, que são produtos de limpeza suaves, que deixam uma fina película de creme hidratante na pele (DESHAYES, 2014).

\section{HIDRATANTE}

Após a limpeza, deve ser aplicado o hidratante, o qual tem função significativa no tratamento da rosácea, pois ajuda a reparar e manter a permeabilidade do estrato córneo (barreira cutânea), aumentando a hidratação da pele, e reduz a possibilidade de irritação cutânea (BALDWIN et al., 2019; ABOKWIDIR; FELDMAN, 2016).

Os principais ativos encontrados em formulações de hidratantes para rosácea são: Alfa-bisabolol que é um derivado do óleo essencial que possui efeito anti-irritante, anti-inflamatório e antimicrobiano; Vitamina E é um poderoso antioxidante que proporciona proteção contra inflamações, acalma a pele e promove o reparo tecidual após lesões; e Alantoína que é conhecida como um dos mais antigos ingredientes ativos anti-inflamatórios (DRAELOS, 2017).

Os hidratantes contêm lipídeos que amaciam e restauram a elasticidade da pele. Os hidratantes atuam na barreira cutânea de diversas maneiras (GONÇALVES, 2016), como apresentado na Tabela 2.

Tabela 2 - Tipos de hidratantes e seus respectivos efeitos na pele

\begin{tabular}{|c|c|c|c|}
\hline \multirow[t]{2}{*}{-} & \multirow[t]{2}{*}{$\because$} & \multicolumn{2}{|r|}{ Exemplos } \\
\hline & & da água & Silicatos, vaselina e derivados de silicone \\
\hline Sullectailues & $\begin{array}{l}\text { uv a } \\
\text { ção }\end{array}$ & $\begin{array}{l}\text { :rna e/ou externa, aumentan- } \\
\text { ¿em um aumento da penetra- } \\
\text { lógicos tópicos }\end{array}$ & Ácido hialurônico e sorbitol \\
\hline Emolientes & $\begin{array}{l}\text { Utili } \\
\text { pree }\end{array}$ & $\begin{array}{l}\text { r a aparência e textura da pele, } \\
\text { entre os corneócitos }\end{array}$ & $\begin{array}{l}\text { Ácidos graxos essenciais, que são encontra- } \\
\text { dos em óleos naturais }\end{array}$ \\
\hline
\end{tabular}

Fonte: Gonçalves (2016).

\section{PROTETOR SOLAR}

O protetor solar é um componente essencial nos cuidados diários da rosácea, visto que existem fortes evidências de que a exposição à luz UV pode provocar sintomas da rosácea, através do aumento de expressão de diferentes quimiocinas inflamatórias, mais especificamente na produção do peptídeo catelicidina LL-37, originando inflamações que consequentemente desencadeiam o eritema e as telangiectasias (ABOKWIDIR; FELDMAN, 2016).

Para pacientes com rosácea é recomendável fazer uso de protetor solar (FPS 30 ou superior), que forneça uma proteção contra os raios UVA e UVB, e ainda contra a radiação de infravermelha (FLOREZ-WHITE, 2019).

Muitos pacientes com rosácea não gostam de usar protetores solares devido a uma sensação de calor na pele. Este calor pode ser devido ao agente ativo do filtro solar que converte a radiação UV em calor. Este é o mecanismo pelo qual todos os filtros solares orgânicos agem. Contudo, protetores solares inorgânicos como dióxido de titânio $\left(\mathrm{TiO}_{2}\right)$ e óxido de zinco ( $\left.\mathrm{ZnO}\right)$, não libertam calor quando atingidos pela radiação UV, mas sim agem por reflexão da energia (DRAELOS, 2017).

\section{MAQUIAGEM CORRETIVA}

A rosácea tem um grande impacto na autoestima do seu portador. Desse modo, a maquiagem corretiva ajuda a restaurar a autoestima desses pacientes. Em suma, os dermatologistas devem encorajar os pacientes com dermatoses faciais a utilizarem maquiagem adequada e segura para melhorar a sua aparência e seu bem-estar (GONÇALVES, 2016). 
As maquiagens corretivas podem ser encontradas em diferentes apresentações: bases fluídas, cremes, cremes compactos, stick's e pós. Estes produtos não devem ser irritantes e alérgicos, e não devem conter perfumes, além de serem não comedogênicos e não acnegênicos, bem como serem clinicamente testados (GONÇALVES, 2016).

Dentre as maquiagens corretivas disponíveis no mercado, pode-se citar: Couvrance ${ }^{\circledR}$ (Avène), Dermablend ${ }^{\circledR}$ (Vichy) e Tolériane ${ }^{\circledR}$ (La Roche Posay). Inclusive, a maquiagem corretiva, possibilita de certo modo uma proteção solar, devido à alta carga de pigmentos contidos em suas formulações. É possível também fazer a neutralização das lesões da rosácea, através do uso do princípio de correção pela cor, que consiste na sobreposição de duas cores. Para neutralização do tom vermelho deve-se utilizar pigmentos verdes (Figura 3) (DESHAYES, 2014).

Figura 3-O princípio da oposição de cores usado na maquiagem corretiva, onde vermelho é neutralizado pelo verde
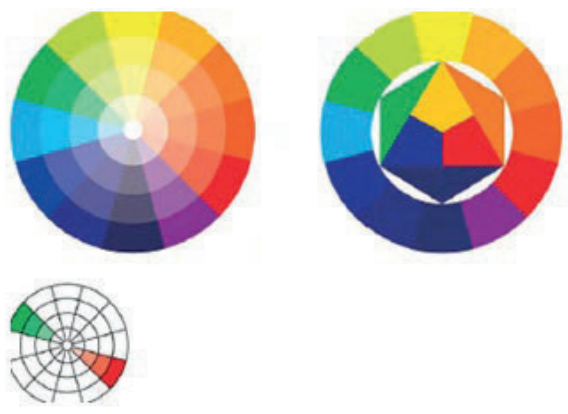

Fonte: Deshayes (2014).

\section{CONCLUSÃO}

Os dermocosméticos se diferenciam do tratamento farmacológico tradicional, por apresentarem uma ausência de risco de eventos adversos e além da possibilidade de serem utilizadas por um período de tempo contínuo e indeterminado, além de oferecerem diversas vantagens a longo prazo, em casos de rosácea leve, ou até como adjuvantes em casos de intensidade moderada a grave.

Os cuidados dermocosméticos devem ser destacados, através de uma rotina de diária de cuidados, desde uma limpeza de pele suave a uma maquiagem corretiva, uma vez que desempenham um papel essencial no controle da doença, trazendo uma melhoria considerável na autoestima e qualidade de vida para os pacientes com rosácea.

Portanto, o tratamento da rosácea é ainda um grande desafio, apesar da grande variedade de opções terapêuticas existentes. Logo, é de suma importância que profissionais habilitados ofereçam informações sobre cuidados de pele e uso das terapias existentes, a fim de otimizar o sucesso terapêutico.

\section{REFERÊNCIAS}

ABOKWIDIR, M.; FELDMAN, S. R. Rosacea Management. Skin Appendage Disorders, [s,I], v. 2, n. 1-2, p. 26-34, 2016.
AGÊNCIA NACIONAL DE VIGILÂNCIA SANITÁRIA, ANVISA, 2015. Resolução da diretoria colegiada - RDC $n^{\circ} \mathbf{0 7}$, de 10 de fevereiro de 2015. Disponível em: http://bvsms.saude.gov.br/bvs/saudelegis/ anvisa/2015/rdc0007_10_02_2015.pdf. Acesso em: 22 nov. 2019.

ARAUJO, T. P. Bioequivalência tópica de produtos contendo metronidazol através da dermatofarmacocinética (DPK). 2016. Dissertação (Mestrado em Ciências Farmacêuticas) - Programa de Pós-Graduação em Ciências Farmacêuticas, Universidade Federal de Pernambuco, Recife.

ASAI, Y. et al. Canadian Clinical Practice Guidelines for Rosacea. J Cutan. Med. Surg., New York, v. 20, n. 5, p. 432-445, 2016.

BALDWIN, H. et al. A novel moisturizer with high sun protection factor improves cutaneous barrier function and the visible appearance of rosacea-prone skin. J. Cosmet. Dermatol., Oxford, v. 18, n. 6, 9. 16861692, 2019.

BUDDENKOTTE, J.; STEINHOFF, M. Recent advances in understanding and managing rosacea. F1000Research, [s.I], v. 7, n. 1, p. 1-10, 2018.

CRIBIER, B. Rosacée : nouveautés pour une meilleure prise en charge. Ann. Dermatol. Vénéréol., Paris, v. 144, n. 8-9, 2017.

DEL ROSSO, J. Q. Management of cutaneous rosacea: emphasis on new medical therapies. Expert Opin.Pharmaco., [s.I], v. 15, n. 14, p. 2029-2038, 2014

DESHAYES, P. Rosacée - prise en charge des patients : hygiène et maquillage. Ann. Dermatol. Vénéréol., Paris, v. 144, n. S2, p. 179-183, 2014.

DRAELOS, Z. D. Cosmeceuticals for rosacea. Clin. Dermatol., Philadelphya, v. 35, n. 2, p. 213-217, 2017

DRENO, B. et al. The science of dermocosmetics and its role in dermatology. J. Eur. Acad. Dermatol. Venereol., Amsterdam, v. 28, n. 11, p. 1409-1417, 2014.

ERDOGAN, H. K. et al. The Evaluation of Contact Sensitivity with Standard and Cosmetic Patch Test Series in Rosacea Patients. Ann. Dermatol., [s,I], v. 30 , n. 3, p. $290-295,2018$.

FLOREZ-WHITE, M. Acne and Rosacea: Special Considerations in the Treatment of Patients With Latin American Ancestry. J. Drugs Dermatol., New York, v. 18, n. 3, p. 124-126, 2019.

GONÇALVES, M. M. B. M. M. Cuidados dermocosméticos da rosácea. 2016. Monografia (Graduação em Farmácia) - Faculdade de Farmácia, Universidade de Coimbra, Portugal, 2016

GONÇALVES, M. M. B. M. M; PINA, M. E. S. R. T. Dermocosmetic care for rosacea. Braz. J. Pharm. Sci., São Paulo, v. 53, n. 4, p. 1-16, 2017.

LUZURIAGA, A. M.; MHLABA, J.; ROMAN, C. Primary Care of Adult Women: Common Dermatologic Conditions. Obstetrics Gynecol. Clin. North Am., Philadelphya, v. 43, n. 2, p. 181-200, 2016.

KARAOSMANOGLU, N.; KARAASLAN, E.; CETINKAYA, P. Evaluation of serum uric acid levels in patients with rosacea. Arch. Dermatol. Res., Berlin, v. 1, n. 1, p. 1-5, 2020.

KRESKEN, J. et al. Dermocosmetics for Use in Rosacea: Guideline of the Society for Dermopharmacy. Arch. Dermatol. Res., Berlin, v. 31, n. 3, p. $147-154,2018$.

SCHALLER, M. et al. Rosacea Management: Update on general measures and topical treatment options. J. Deutsch. Dermatol. Ges., Berlin, v. 14, n. 6, p. 17-27, 2016.

SCHMUTZ, J. L. Signes cliniques de la rosacée. Ann. dermatol. vénéréol. Paris, v. 14, n. 1, p. 151-157, 2014. 
SIDDIQUI, K.; GOLD, L. S.; GILL, J. The efficacy, safety, and tolerability of ivermectin compared with current topical treatments for the inflammatory lesions of rosacea: a network meta-analysis. SpringerPlus, [s.l],v. 5, n. 1, 22, p. 1511, 2016.

SOCIEDADE BRASILEIRA DE DERMATOLOGIA (SBD). 2018. Rosácea.
Disponível em: https://www.sbd.org.br/dermatologia/pele/doencaseproblemas/rosacea/62/. Acesso em: 01 nov. 2019

VAN ZUUREN, E. J. Rosacea. N. Engl. J. Med., Boston, v. 377, n. 18, p. 1754-1764, 2017.

Submetido em: $20 / 02 / 2020$

Aceito em: 23/06/2020 